\title{
Avaliação do tratamento específico para o Trypanosoma cruzi em crianças, na evolução da fase indeterminada
}

\author{
Specific treatment evaluation for Trypanosoma cruzi in children, \\ in the evolution of the indeterminate phase
}

Celeste A.N. Silveira, Edwin Castillo e Cleudson Castro

\begin{abstract}
Resumo Doze pacientes com idades entre 7 a 12 anos, na forma indeterminada da doença de Chagas, com sorologia e xenodiagnóstico positivos, receberam tratamento específico. Dois pacientes tomaram $7 \mathrm{mg} / \mathrm{kg}$ de nifurtimox durante 60 e 90 dias e 10 usaram $5-7 \mathrm{mg} / \mathrm{kg}$ de benznidazol durante 60 dias. A evolução clínica foi verificada através de exame clínico, eletrocardiograma, exame radiológico contrastado do esôfago. Após o tratamento somente uma $(8,3 \%)$ paciente apresentou todos os exames negativos. Oito deles foram avaliados após oito anos do tratamento e 4 acompanhados durante 20 anos. Sete $(58,4 \%)$ permaneceram na forma indeterminada e $4(33,3 \%)$ chagásicos progrediram clinicamente para cardiopatia grau II e/ou esofagopatia, apesar do tratamento precoce. São necessários estudos com maior número de crianças na fase indeterminada e acompanhamento a longo prazo para se estabelecer a influência do tratamento específico na evolução da doença de Chagas.
\end{abstract}

Palavras chaves: Doença de Chagas. Fase indeterminada. Tratamento específico. Evolução clínica.

Abstract Twelve chagasic patients between the ages of seven and twelve, in the indeterminate phase with serology and xenodiagnosis positive, received the specific treatment. Eight of these were evaluated after an eight-year treatment period and four were followed-up during 20 years. Two patients took $7 \mathrm{mg} / \mathrm{kg}$ of nifurtimox during sixty and ninety days and ten of these used $5-7 \mathrm{mg} / \mathrm{kg}$ of benznidazole during 60 days. The clinical outcome was verified through clinical examination, electrocardiogram and contrasted X-ray of the esophagus. After the treatment, only one patient presented negativity in all the examinations. Seven $(58.4 \%)$ remained in the indeterminate form and despite the precocious treatment four chagasic patients (33.3\%) progressed clinically to second degree cardiopathy and/or megaesophagus.

Key-words: Chagas' disease. Indeterminate phase. Specific treatment. Clinic evolution.

A quimioterapia da doença de Chagas continua sendo um desafio. Atualmente, apenas duas drogas nitrohetrocíclicas são usadas, sendo que no Brasil só está disponível o benznidazol.

A Fundação Nacional de Saúde ${ }^{11}$, considerando a experiência acumulada por vários pesquisadores no tratamento dessa doença ${ }^{16915}{ }^{16}$, promoveu uma reunião de grupo de assessores que recomendou o tratamento da fase aguda e da fase crônica inicial em crianças.
Alguns autores ${ }^{1691516}$ têm relatado bons resultados no tratamento da doença de Chagas com benznidazol em crianças abaixo de 12 anos mostrando a negativação da sorologia. Contudo o tempo de acompanhamento pós tratamento dessas crianças tem sido curto para avaliar a evolução da forma indeterminada.

O objetivo deste trabalho é o de avaliar a evolução de crianças tratadas com nifurtimox e benznidazol após 8 e 20 anos de seguimento.

Núcleo de Medicina Tropical e Nutrição da Universidade de Brasília, Brasília, DF, Brasil.

Endereço para correspondência: Dra. Celeste A.N. Silveira. Núcleo de Medicina Tropical. Caixa Postal 4517, 70919-970 Brasília, DF. Recebido para publicação em 13/10/99. 


\section{MATERIAL E MÉTODOS}

Foram tratadas 12 crianças de ambos os sexos, residentes em Mambaí, Goiás, área endêmica de doença de Chagas, onde a transmissão está interrompida há mais de 10 anos. As idades dos pacientes à época do tratamento variaram de 7 a 12 anos. Todas as crianças apresentavam no mínimo duas sorologias para doença de Chagas (fixação do complemento, imunofluorescência, hemaglutinação e/ou imunoenzimático de ELISA) reagentes, realizadas em dois laboratórios de referência. A confirmação parasitológica foi feita através de xenodiagóstico, exceto em uma criança cujo exame foi negativo. O exame clínico e eletrocardiograma foram normais.

A autorização para o tratamento foi fornecida pelos pais ou responsáveis.

Os medicamentos usados foram: nifurtimox, $7-8 \mathrm{mg} / \mathrm{kg} / \mathrm{dia}$ durante $60-90$ dias em dois pacientes e benznidazol, $5-7 \mathrm{mg} / \mathrm{kg} / \mathrm{dia}$ durante 60 dias, nos demais. Os pacientes foram acompanhados pelos autores em consultas clínicas ambulatoriais semanais, onde era fornecido medicamento para 7 dias e a administração da medicação foi supervisionada pelo responsável.
Quatro crianças foram tratadas em 1978 e oito em 1989. Após o tratamento, ao longo destes anos, foram realizadas avaliações clínicas e laboratoriais, eletrocardiogramas, $\mathrm{Rx}$ contrastado do esôfago e vários xenodiagnósticos. A última avaliação (1997/98) constou de exame clínico, sorologias convencionais (HI, TIF e ELISA), PCR, xenodiagnóstico e ECG. Para cada xenodiagnóstico utilizou-se 40 ninfas do $1^{\circ}$ estádio de Dipetalogaster maximus e posteriormente examinado o conteúdo intestinal dos insetos com 40 dias, no Laboratório de Xenodiagnóstico do Núcleo de Medicina Tropical/UnB. Para realização do PCR foi colhido $10 \mathrm{ml}$ de sangue venoso e colocado em tubo com igual volume de $6 \mathrm{M}$ Hidroclorado de Guanidina e 200mM solução de EDTA, e encaminhado ao Laboratório de Biologia Molecular do Instituto Oswaldo Cruz no Rio de Janeiro. As sorologias convencionais foram realizadas no Laboratório de Parasitologia do Instituto de Patologia Tropical em Goiânia. Foram considerados curados os pacientes que na última avaliação apresentaram os teste sorológicos, PCR e xenodiagnósticos negativos.

\section{RESULTADOS}

Os 12 pacientes à época do tratamento apresentavam doença de Chagas crônica indeterminada provavelmente com infecção recente, já que todos tinham entre 7 a 12 anos de idade e, foram diagnósticados pela presença de anticorpos IgG específicos. Em um deles (5548), foi possível, retrospectivamente, identificar os sinais e sintomas da fase aguda aos seis meses de vida. Segundo o relato da mãe, a criança apresentou febre, edema ocular e presença do triatomíneo no leito, porém não procurou assistência médica.

Onze pacientes tinham xenos positivos antes do início da terapêutica. A paciente (5364) cujos dois xenos prévios ao tratamento foram negativos, repetiu os teste sorológicos convencionais (IFI, HAI, ELISA) e aglutinação direta com 2 mercaptoetanol (AD-2ME) confirmando a infecção chagásica. Todos os chagásicos encontravamse na forma indeterminada, sem alterações clínicas, radiológicas e eletrocardiográficas. Os quatro pacientes tratados em 1978, dois com nifurtimox e dois com benznidazol, tiveram acompanhamento prospectivo de 20 anos. Os demais receberam benznidazol em 1989 e foram acompanhados por oito anos. Dois chagásicos (5494 e 5504) tomaram o benznidazol por 52 e 58 dias respectivamente, os demais por 60 dias. As idades atuais variaram de 16 a 31 anos, apresentando duas modas 17 e 31 anos (Tabela 1).

A cura foi observada em 1 (8,3\%) paciente, levando em consideração a negativação sorológica, dos xenodiagnósticos e PCR. A persistência da positividade em todas as técnicas sorológicas após terapêutica foi observada em 4 (33,3\%) dos pacientes. Mesmo levando em conta as diferenças metodológicas e temporais em 7 $(58,4 \%)$ houve redução dos títulos, pois estes chagásicos passaram a apresentar resultados duvidosos ou negativos na mesma amostra de sangue por diferentes técnicas (Tabela 2).

A evolução clínica após a terapêutica foi verificada através de exame clínico, eletrocardiogramas e em quatro chagásicos, exames radiológicos contrastados do esôfago. Nenhum paciente apresentou história de obstipação e foram considerados sem colopatia (Tabelas 3 e 4$)$. Sete $(58,4 \%)$ permaneceram na forma indeterminada $1(8,3 \%)$ apresentou BIRD, 
não sendo considerado como cardiopatia chagásica e $4(33,3 \%)$ pacientes progrediram clinicamente para cardiopatia grau II e/ou esofagopatia, apesar do tratamento específico precoce.

Tabela 1 - Distribuição por sexo, idade à época do tratamento e atual, medicação usada e tempo de evolução dos 12 portadores de T. cruzi estudados em Mambaí, G0, 1998.

\begin{tabular}{|c|c|c|c|c|c|}
\hline Registro & Sexo & Idade à época do tratamento & Idade atual & Medicação usada & Evolução (anos) \\
\hline 348 & $\mathrm{M}$ & 11 & 31 & nifurtimox & 20 \\
\hline 1004 & M & 11 & 29 & benznidazol & 20 \\
\hline 1281 & M & 12 & 31 & benznidazol & 20 \\
\hline 4161 & $\mathrm{~F}$ & 12 & 31 & nifurtimox & 20 \\
\hline 4746 & M & 11 & 19 & benznidazol & 8 \\
\hline 5155 & M & 12 & 21 & benznidazol & 8 \\
\hline 5230 & $\mathrm{~F}$ & 8 & 17 & benznidazol & 8 \\
\hline 5334 & $\mathrm{~F}$ & 8 & 16 & benznidazol & 8 \\
\hline 5364 & $\mathrm{~F}$ & 9 & 17 & benznidazol & 8 \\
\hline 5494 & $\mathrm{~F}$ & 7 & 16 & benznidazol & 8 \\
\hline 5504 & M & 12 & 20 & benznidazol & 8 \\
\hline 5548 & $M$ & 9 & 17 & benznidazol & 8 \\
\hline
\end{tabular}

Tabela 2 - Resultados das reações sorológicas, por diferentes técnicas, xenodiagnóstico pré e pós tratamento e PCR pós tratamento dos portadores de T. cruzi estudados em Mambaí, GO, 1998.

\begin{tabular}{|c|c|c|c|c|c|c|c|c|c|c|c|}
\hline \multirow{3}{*}{ Reg } & \multicolumn{6}{|c|}{ Sorologias } & \multicolumn{4}{|c|}{ Xenodiagnóstico } & \multirow{3}{*}{$\begin{array}{c}\text { PCR } \\
1997 / 98\end{array}$} \\
\hline & \multicolumn{3}{|c|}{ pré } & \multicolumn{3}{|c|}{ pós } & \multirow[t]{2}{*}{ pré } & \multicolumn{3}{|c|}{ pós } & \\
\hline & $\mathrm{FC}$ & IFI & $\mathrm{HAl}$ & IFI & $\mathrm{HAl}$ & ELISA & & $1978 / 79$ & $1984 / 89$ & $1997 / 98$ & \\
\hline $348^{*}$ & pos & pos & pos & duv & pos & pos & pos & neg neg & neg neg & neg & neg \\
\hline $1004^{*}$ & pos & pos & pos & duv & duv & pos & pos & neg neg & neg neg & neg & neg \\
\hline $1281^{*}$ & pos & pos & pos & duv & neg & duv & pos & neg neg & neg neg & neg neg & neg \\
\hline $4161^{*}$ & pos & pos & pos & pos & pos & pos & pos & neg neg & neg & neg & neg \\
\hline 4746 & pos & pos & pos & pos & pos & pos & pos & - & - & neg pos & pos \\
\hline 5155 & pos & pos & pos & pos & pos & pos & pos & - & - & neg neg & neg \\
\hline 5230 & pos & pos & pos & pos & pos & pos & pos & - & - & neg & neg \\
\hline 5334 & pos & pos & pos & duv & duv & pos & pos & - & - & neg neg neg & neg \\
\hline 5364 & pos & pos & pos & neg & neg & neg & neg & - & - & neg neg & neg \\
\hline 5494 & pos & pos & pos & duv & neg & pos & pos & - & - & neg neg & neg \\
\hline 5504 & pos & pos & pos & pos & duv & pos & pos & - & - & neg neg & neg \\
\hline 5548 & pos & pos & pos & duv & neg & pos & pos & - & - & neg & neg \\
\hline
\end{tabular}

duv: duvidoso; pos: positivo; neg: negativo. FC: fixação do complemento; HAl: hemaglutinação indireta; IFI: imunofluorescência indireta; ELISA: imunoenzimático.

* Os exames pré tratamento dos quatro primeiros pacientes foram realizados em 1975, e dos demais em 1989.

Tabela 3 - Resultados dos exames eletrocardiográficos dos 12 portadores de T. cruzi estudados em Mambaí, GO, por sexo, idade, pré e pós tratamento, 1998.

\begin{tabular}{|c|c|c|c|c|c|c|}
\hline \multirow{3}{*}{ Reg. } & \multirow{3}{*}{ Sexo } & \multirow{3}{*}{ Idade } & \multicolumn{4}{|c|}{ Eletrocardiograma } \\
\hline & & & \multicolumn{2}{|c|}{ pré } & \multicolumn{2}{|c|}{ pós } \\
\hline & & & $75 / 89$ & 1982 & 1988 & 1998 \\
\hline $348^{*}$ & $\mathrm{M}$ & 31 & normal & normal & Rítmo juncional & normal \\
\hline $1004^{*}$ & $M$ & 29 & normal & normal & $\mathrm{BIRD} / \mathrm{BAV} 1^{\circ} \mathrm{G}$ & $\mathrm{BCRD} / \mathrm{BAV} 1^{\circ} \mathrm{G}$ \\
\hline $1281^{*}$ & M & 31 & normal & normal & normal & normal \\
\hline $4161^{*}$ & $\mathrm{~F}$ & 31 & normal & - & - & normal \\
\hline 4746 & M & 19 & normal & - & - & BCRD \\
\hline 5155 & M & 21 & normal & - & - & BCRD \\
\hline 5230 & $\mathrm{~F}$ & 17 & normal & - & - & normal \\
\hline 5334 & $\mathrm{~F}$ & 16 & normal & - & - & normal \\
\hline 5364 & $\mathrm{~F}$ & 17 & normal & - & - & normal \\
\hline 5494 & $\mathrm{~F}$ & 16 & normal & - & - & normal \\
\hline 5504 & M & 20 & normal & - & - & BIRD \\
\hline 5548 & M & 17 & normal & - & - & normal \\
\hline
\end{tabular}

* Os exames pré tratamento foram realizados nos quatro primeiros pacientes em 1975 ; nos demais em 1989. 
Tabela 4 - Distribuição dos 12 portadores de T. cruzi estudados em Mambaí, GO, segundo as formas clínicas pré e pós tratamento, 1998.

\begin{tabular}{|c|c|c|c|c|c|c|c|c|c|c|c|c|}
\hline \multirow{3}{*}{ Reg } & \multicolumn{4}{|c|}{ Esofagopatia } & \multicolumn{4}{|c|}{ Colopatia } & \multicolumn{4}{|c|}{ Cardiopatia } \\
\hline & \multicolumn{2}{|c|}{ pré } & \multicolumn{2}{|c|}{ pós } & \multicolumn{2}{|c|}{ pré } & \multicolumn{2}{|c|}{ pós } & \multicolumn{2}{|c|}{ pré } & \multicolumn{2}{|c|}{ pós } \\
\hline & $75 / 89$ & 1982 & 1988 & 1998 & $75 / 89$ & 1982 & 1988 & 1998 & $75 / 89$ & 1982 & 1988 & 1998 \\
\hline $384^{*}$ & aus & - & aus & aus & aus & aus & aus & aus & aus & aus & $\mathrm{Cl}$ & aus \\
\hline $1004^{*}$ & aus & - & aus & aus & aus & aus & aus & aus & aus & aus & $\mathrm{Cll}$ & Cll \\
\hline $1281^{*}$ & aus & aus & $\mathrm{Gl}$ & GI & aus & aus & aus & aus & aus & aus & aus & aus \\
\hline $4161^{*}$ & aus & - & - & aus & aus & - & - & aus & aus & - & - & aus \\
\hline 4746 & aus & - & - & aus & aus & - & - & aus & aus & - & - & CII \\
\hline 5155 & aus & - & - & GI & aus & - & - & aus & aus & - & - & CII \\
\hline 5230 & aus & - & - & aus & aus & - & - & aus & aus & - & - & aus \\
\hline 5334 & aus & - & - & aus & aus & - & - & aus & aus & - & - & aus \\
\hline 5364 & aus & - & - & aus & aus & - & - & aus & aus & - & - & aus \\
\hline 5494 & aus & - & - & aus & aus & - & - & aus & aus & - & - & aus \\
\hline 5548 & aus & - & - & aus & aus & - & - & aus & aus & - & - & aus \\
\hline
\end{tabular}

Aus : ausência; GI : megaesôfago grau I; Cl : cardiopatia grau I; CIl: cardiopatia grau II.

* Obs. Os exames pré tratamento foram realizados nos quatro primeiros pacientes, em 1975 e nos demais em 1989.

\section{DISCUSSÃO}

Os nitrohetrocíclicos atuam contra o T. cruzi e são capazes de curar doentes na fase aguda em 70 a $100 \%$ enquanto na fase crônica indeterminada as percentagens variam entre 6 a $30 \%$. A falha terapêutica observada em parte dos pacientes chagásicos agudos pode ser explicada pela existência de cepas naturalmente resistentes às drogas ${ }^{4}$. No entanto, ainda não se tem uma explicação adequada para os baixos índices de resposta na fase crônica.

Como critérios de cura são utilizados xenodiagnósticos e hemoculturas mesmo reconhecendo-se a baixa sensibilidade destes exames. Recentemente, vários trabalhos têm sido publicados $^{520}$ usando a técnica de polimerase em cadeia (PCR) para detecção do T. cruzi, apresentando alta sensibilidade entre 96,5 a $100 \%$. A influência da terapêutica específica sobre a parasitemia é evidente, traduzida pela negatividade persistente dos exames parasitológicos, exceto em um paciente cujo xeno voltou a positivar.

A persistência da positividade da sorologia na grande maioria dos pacientes tratados na fase crônica tem levado alguns autores ${ }^{17}$ a considerá-la como memória imunológica, com a manutenção de anticorpos na ausência do T. cruzi. Andrade et al ${ }^{23}$ demonstraram, através de imunoeletromicroscopia que antígenos parasitários retidos nas células dendríticas do baço podem ser responsáveis por esta memória imunológica, capaz de manter o organismo em processo de estimulação antigênica.

O sucesso terapêutico para doença de Chagas é considerado quando há negativação parasitológica e sorológica após o tratamento específico ${ }^{19}$.

O principal efeito que se busca com o tratamento antiparasitário é a cura, mas muitas vezes este objetivo não é alcançado. Contudo, se a progressão da doença puder ser evitada a terapêutica se justificaria.

Neste trabalho, apenas em 1 paciente a cura foi observada.

Há dúvidas se o tratamento na forma indeterminada tardia é capaz de interferir na evolução da doença. Estudos longitudinais em áreas endêmicas têm demonstrado que anualmente $2 \%$ dos pacientes na forma indeterminada evoluem para alguma forma clínica ${ }^{13}$ e $0,3 \%$ para megaesôfago ${ }^{7}$.

Macêdo e Silveira ${ }^{14}$, comparando a evolução de chagásicos na forma indeterminada tratados com tripanosomicidas e placebo por um período de cinco anos, não encontraram diferença na evolução dos dois grupos. Resultados semelhantes foram relatados por Estani et al ${ }^{8}$ em crianças com infecção recente, tratadas com benznidazol e grupo placebo e avaliadas quatro anos após a terapêutica. Contudo, Fragatta Filho et $\mathrm{al}^{10} \mathrm{e}$ Viotti et $\mathrm{al}^{18}$ acompanhando chagásicos crônicos por um período médio de 8 anos, mostraram piora clínica e eletrocardiográfica entre os não tratados quando comparados aos tratados.

Neste estudo, 4 (33,3\%) pacientes, todos do sexo masculino, evoluíram para alguma forma clínica. Três deles para a forma cardíaca grau II, 
segundo critério de Macêdo ${ }^{12}$, e 2 pacientes desenvolveram esofagopatia.

Embora com pequeno número de casos, a evolução clínica dos quatro pacientes para as formas cardíacas e/ou digestivas, denotam que o tratamento não impediu a progressão da doença de Chagas. São necessários estudos com maior número de crianças na forma indeterminada e acompanhamento a longo prazo para se estabelecer a influência do tratamento específico na evolução da doença de Chagas.

\section{AGRADECIMENTOS}

Agradecemos ao Prof. Alejandro Luquetti Ostermayer e as biomédicas Suelene Brito $\mathrm{N}$. Tavares e Rosângela Amaral de Oliveira, do Instituto de Patologia Tropical - UFG, pela realização das sorologias para doença de Chagas e aos Drs. Octavio Fernandes e Constança Britto, do Laboratório de Biologia Molecular da FIOCRUZ, RJ, pela realização do PCR.

\section{REFERÊNCIAS BIBLIOGRÁFICAS}

1. Andrade AL, Zicker F, Oliveira RM, Silva SA, Luquetti A, Travassos LR, Almeida IC, Andrade SS, Andrade JG, Martelli CMT. Randomised trial of efficacy of benznidazole in treatment of early Trypanosoma cruzi infection. Lancet 348:1407-1413, 1996.

2. Andrade SG, Freitas LA, Peyrol S, Pimentel AR, Sandigursky M. Trypanosoma cruzi antigens detected by immunoelectron microscopy in the spleen of the mice serologically positive but parasitologically cured chemotherapy. Revista da Sociedade Brasileira de Medicina Tropical 21:41-42, 1988.

3. Andrade SG, Freitas LA, Peyrol S, Pimentel AR, Sandigursky M. Experimental chemotherapy of Trypanosoma cruzi infection: persistence of parasite antigens and positive serology in parasitologically cured mice. Bulletin of the World Organization 69:191197,1991.

4. Andrade SG, Rassi A, Magalhães JB, Ferriolli Filho F, Luquetti AO. Specific chemotherapy of Chagas disease: a comparison between the response in patients and experimental animals inoculated with the same strains. Transactions of the Royal Society of Tropical Medicine and Hygiene 86:624-626, 1992.

5. Avila HA, Pereira JB, Thieman D, Paiva E, Degrave W, Morel CM, Simpson L. Detection of Trypanosoma cruzi in blood specimens of chronic chagasic patients by polymerase chain reaction amplification of kinetoplast minicircle DNA: comparison with serology and xenodiagnosis. Journal of Clinical Micobiology 31:24212426, 1993

6. Cançado JR. Terapêutica específica In: Dias JCP, Coura JR (eds) Clínica e terapêutica de doença de Chagas 32351, 1997.

7. Castro C, Macêdo VO, Rezende JM, Prata A. Estudo radiológico longitudinal do esôfago em área endêmica da doença de Chagas, em um período de 13 anos. Revista da Sociedade Brasileira de Medicina Tropical 27:227-233, 1994.
8. Estani SS, Segura EL, Ruiz AM, Velazquez E, Porcel BM, Yampotis C. Efficacy of chemotherapy with benznidazole in children in the indeterminate phase of Chagas'disease. The American Journal Tropical Medicine and Hygiene 59:526-529, 1998.

9. Ferreira HO. Tratamento da forma indeterminada da doença de Chagas com Nifurtimox e Benzonidazol. Revista da Sociedade Brasileira de Medicina Tropical 23:209-211, 1990.

10. Fragata Filho AA, Silva MAD, Boainain E. Tratamento etiológico da doença de Chagas na fase aguda e crônica. Revista da Sociedade de Cardiologia do Estado São Paulo 4:192-97, 1994.

11. Ministério da Saúde. Tratamento etiológico da doença de Chagas, $2^{\natural}$ edição, Fundação Nacional da Saúde, 1997.

12. Macêdo V. Influência da exposição à reinfecção na evolução da doença de Chagas. Revista de Patologia Tropical 5:33-116, 1976.

13. Macêdo V. Forma indeterminada da doença de Chagas. Jornal Brasileiro de Medicina 38:34-40, 1980.

14. Macêdo V, Silveira CAN. Perspectiva da terapêutica na doença de Chagas. Experiência na forma indeterminada. Revista da Sociedade Brasileira de Medicina Tropical 20(supl II):24-26, 1987.

15. Prata A. Tratamento da doença de Chagas pelo Nifurtimox (Bayer 2502). Revista da Sociedade Brasileira de Medicina Tropical 9:297-307, 1975.

16. Prata A, Ferreira H. Dois casos de doença de Chagas aguda aparentemente curados pelo Nitrofurazona. Gazeta Médica da Bahia 69:25-29, 1969.

17. Schenone H, Concha L, Aranda R. Experiência terapêutica com el bayer 2502 en la infeccion chagásica cronica del adulto. Importance del uso adecuado del xenodiagnóstico. Boletim Chileno de Parasitologia 24:6669, 1969.

18. Viotti R, Vigliano C, Armenti H, Segura E. Treatment of chronic Chagas' disease with benznidazole: clinical and 
serologic evolution of patient with log-term follow-up. American Heart Journal 127:151-162, 1994.

19. World Health Organization. Control of Chagas' disease. WHO Technical Report 811, Geneva, 1991.

20. Wincker P, Britto C, Pereira JB, Cardoso MA, Aelemann $\mathrm{W}$, Morel CM. Use of a simplified polymerase chain reaction procedure to detect in blood sample from chronic chagasic patient in a rural endemic area. American Journal of Tropical Medicine and Hygiene 5:771-777, 1994. 